\title{
Using an online CBT-based intervention to improve academic performance in students with low mood: A pre-post study with historical control
}

Suheir Awadalla ( $\sim$ suheir.awadalla@nottingham.ac.uk)

University of Nottingham School of Medicine https://orcid.org/0000-0002-6178-9522

Bethan Davies

University of Nottingham

Cristine Glazebrook

University of Nottingham

Research article

Keywords: academic performance, MoodGYM, depression, anxiety, online intervention

Posted Date: February 12th, 2021

DOI: https://doi.org/10.21203/rs.3.rs-228617/v1

License: (c) (i) This work is licensed under a Creative Commons Attribution 4.0 International License.

Read Full License 


\section{Abstract \\ Background}

Online CBT-based interventions have shown potential to improve mental health in university students. However, their impacts in West Asian cultures and on educational achievement have yet to be fully investigated. This study aims to explore the feasibility, acceptability and potential effectiveness of a selfdirected, internet-delivered, cognitive-behavioural skills training program (MoodGYM) in reducing depression and improving academic performance in university students in the United Arab Emirates (UAE).

\section{Methods}

This exploratory pre-post intervention study with an historic control group recruited 50 students from one UAE university, with GPA $<2$ and self-reporting at least one of two key depressive symptoms. Preintervention, participants completed an online survey including most recent grade point average (GPA), number of attendance warnings and the Hospital Anxiety and Depression Scale (HADS). Participants were then sent a link to MoodGYM. After eight weeks, 44 participants repeated the survey and provided feedback on MoodGYM ( $88 \%$ follow-up). A subgroup of 19 students with GPA scores $<2$ at baseline formed an historical control group for change in GPA and attendance warnings.

\section{Results}

Total HADS-Depression scores fell at post intervention $(P=0.004)$ and the proportion of participants scoring above the cut-off for depression (HADS-D $\geq 8)$ fell from $77.2-27.3 \%(p<0.001)$. There was also a substantial fall in HADS-Anxiety scores $(p<0.001)$ and the proportion of participants above the cut-off for anxiety (HADS-A $\geq 8)$ fell from $50 \%$ to $11.4 \%(p=0.001)$. GPA scores improved substantially over time ( $p$ $<0.001, d=1.3)$ and attendance warnings reduced $(p=0.008, d=0.6)$. Compared to historic control, the intervention group had higher GPA at follow-up $(p<0.030 d=0.6)$ fewer attendance warnings $(p=0.036 d$ $=0.7)$. Most students $(79.6 \%)$ evaluated MoodGYM as useful and all students completed at least 2 MoodGYM modules. More modules completed $(p=0.005)$ and greater reduction in attendance warnings $(p=0.007)$ were independently associated with greater improvement in GPA scores at follow-up.

\section{Conclusions}

This study provides support for a web based mental health promotion intervention (MoodGYM) to improve academic achievement in university students with depressive symptoms. Further research is needed to explore how MoodGYM can be best implemented within University settings. 


\section{Background}

Studies indicate a high prevalence of mental health problems, such as depression, among university students compared to their non-student peers [1]. This is concerning, as depression has been shown to impact all areas of student well-being, including academic achievement. Students with depressive symptoms tend to have poor classroom engagement, peer interactions, and attendance [2]. Thus, depression negatively influences academic progress and encourages under-achievement among university students.

A recent representative survey [3] reported that over one-third of Emirati students at one university scored above the threshold for major depressive disorder. Depressive symptoms at baseline, but not anxiety symptoms, were shown to predict academic performance at follow-up, as measured by grade point average (GPA). This supports previous evidence that depression negatively impacts university students' academic performance [4] and suggests that an intervention to help students manage low mood could reduce this educational disadvantage.

In many Arab countries, established mental health resources are limited, and negative beliefs about mental health care can act as a barrier to engaging in help-seeking behaviour [5]. Therefore, online therapeutic interventions offer considerable advantages in terms of student access and privacy. Existing research suggests that technology-based interventions could fill the gap between the need for and access to mental health services among university students [6]. There is a growing need to create supportive environments for students who may experience emotional difficulties during university life [7]. 'Internetdelivered technology' in counselling refers to psychological online interventions provided by utilising various multimedia formats and interactive features to engage users and promote intervention effectiveness [8]. Benefits of internet-delivered interventions include customisation to student needs, anonymous access, and a more comfortable private setting in which to access sensitive information [6]. Recently, these advantages have led to increased focus on the use of online interventions, resulting in several studies that examined such programs. [9].

It is known that online interventions can effectively improve university students' mental health $[10,11]$; however, their impact on educational attainment has yet to be fully explored. In 2020, Bolinski and colleagues [12] conducted a systematic review to evaluate the impact of online mental health interventions on academic performance. Meta-analysis of the six RCT studies to include academic performance as an outcome showed beneficial effects for depression but revealed only a small nonsignificant effect for academic achievement. None of the six studies to address mental health and academic performance targeted students who had symptoms of depression and struggled academically at baseline.

Cognitive behavioural therapy (CBT) is a collaborative therapy that focuses on how a person's thoughts, beliefs, and attitudes affect their emotions and behaviours [13]. CBT is recommended as a treatment for mild to moderate depression and has been shown to be effective [14]; however, limited accessparticularly in middle- and low-income countries-and stigma associated with poor mental health, may 
hinder access to physical treatment resources [15]. Thus, cost-effective and widely accessible alternatives to in-person treatment are required. Adolescents use technology at high rates [16], yet innovative methods of online treatment have not fully exploited students' expertise. For example, despite evidence confirming the effectiveness of computerised CBT for reducing depressive and anxiety symptoms, to our knowledge, no study has evaluated a CBT-based online intervention targeting students with depressive symptoms who are also struggling academically.

MoodGYM (https://moodgym.anu.edu.au/welcome/faq) is an online CBT-based program designed to prevent symptoms of emotional distress and various mental health disorders in adolescents, which has shown promise in Australian studies [17]. MoodGYM comprises five modules consisting of written information, animations, interactive exercises, and quizzes designed to teach skills known to prevent depression and anxiety among young people $[18,19]$.

Research has investigated the effects MoodGYM across a variety of settings and using different study designs. Twomey and O'Reilly [20] conducted a systematic review of 11 studies to evaluate the effectiveness of MoodGYM in reducing depressive symptoms and general psychological distress in adults. They found that studies with no treatment controls, face-to-face guidance, and high adherence to MoodGYM modules revealed a more positive effect on symptoms of depression and other psychological stress. Furthermore, a stronger effect was found in studies conducted in Australia compared with Europe. The study concluded that MoodGYM could provide primary support to participants with mental health issues, considering that adherence rates and cross-cultural factors may affect the influence of CBT webbased programs.

There is some evidence that MoodGYM may be more effective where the intervention is targeted, Christensen and colleagues [21] found that in people with baseline levels of anxiety and depression who accessed MoodGYM, their symptoms significantly decreased after completing the program. Canadian university students at risk of depression randomised to MoodGYM showed greater reduction in depressive symptoms and were significantly less likely to be diagnosed with a major depressive disorder at 4-month follow-up compared to attentional control [22].

This study aims to evaluate the feasibility, acceptability and effectiveness of targeting MoodGYM at academically struggling Emirati students with symptoms of depression.

\section{Objectives}

The objectives of the present study were as follows:

- To explore the acceptability and feasibility of using MoodGYM to improve academic achievement in university students with symptoms of low mood and poor academic performance in the UAE.

- To investigate the potential effectiveness of a self-directed, internet-delivered cognitive-behavioural skills therapy (MoodGYM) in improving academic performance (GPA) and mood in university students in the UAE with poor academic performance. 
- To investigate the relationship between Moodgym uptake and improvement in GPA at postintervention.

\section{Methods}

\section{Design}

This study used a pre-post pilot design with an historical control group to evaluate changes in GPA. Data were collected via online surveys administered at baseline and follow-up two months after using MoodGYM.

\section{Participants and recruitment}

Participants were undergraduate students at a public university in the UAE, aged 18 and over. Students were selected from two campuses (Dubai and Abu-Dhabi), and recruited through their academic advisors. The target recruitment was 50 participants.

The target sample size was 50 male and female undergraduate students who met the following inclusion criteria:

- Undergraduate students in their second, third, or fourth year of study at one university in the UAE

- Scheduled to attend an academic advisory seminar to address poor academic performance (GPA less than 2.0)

- Aged 18 years or over

- Self-identified as having at least one of two key symptoms of low mood,[23]

\section{Historical control group}

Students from a previous longitudinal cohort study of UAE university students [3] were selected as the comparison group, if they had a GPA $<2.0$ at baseline and a GPA score reported at two-month follow-up. The control group students $(n=19)$ had similar GPAs, attendance warnings, and demographic characteristics at baseline as the intervention group ( $n=44)$. This group (no intervention) was used as a comparison group to evaluate the academic improvement in the intervention group.

\section{Power calculation}

With a historic control group of $n=19$ and assuming a $70 \%$ response rate at follow-up in the intervention group (35/50), it was estimated that the study would have $90 \%$ power to detect 1 SD difference $(d=1)$ in GPA between groups at follow-up with a probability of a type 1 error $<0.05$ [24].

\section{Ethical approval}


Ethical approval was obtained from the Division of Psychiatry and Applied Psychology Ethics Subcommittee (reference number: 0397) and the Research Ethics Committee at Zayed University (ref ZU19_46_F). Participation was voluntary. Students provided the written consent online to participate in the two anonymous online surveys, which were linked by self-generated identifiers.

\section{Measures}

\section{Mental health}

The Hospital Anxiety and Depression Scale (HADS) is a self-assessment tool developed to detect states of depression and anxiety in non-psychiatric settings [25]. The HADS consists of 14 items, with 7 assessing anxiety (HADS-A) and 7 assessing depression (HADS-D). For both subscales, scores of $\leq 7$ indicate non-cases, while scores of 8-10, 11-14, and 15-21 indicate mild, moderate, and severe depression and anxiety respectively [26]. Cronbach's alpha ranged from 0.68 to 0.93 (mean $=0.83$ ) for the HADS-A and from 0.67 to 0.90 (mean $=0.82$ ) for the HADS-D; [27]. The optimal cut off for anxiety caseness is HADS-A $\geq 8$ (sensitivity 0.89 , specificity 0.78 ) and for depression caseness HADS-D $\geq 8$ (sensitivity 0.83 and specificity 0.79 [27]. The HADS has been validated in many languages, countries, and settings, including with university students [28]. An Arabic version of the HADS has been validated in Saudi Arabia [29], Kuwait [30], and the UAE [31] in primary-care settings and, recently, in hospitalised patients [32]. Since UAE students study in English, the English language version was used in the present study.

\section{Academic performance}

Participants reported their most recent GPA and the number of academic warnings about poor attendance they had received during the last semester. Possible GPA scores range from 0-4, with higher scores indicating better academic performance. In the present study, a GPA below 2.0 was considered a sign of academic difficulties.

\section{Intervention}

MoodGYM is an internet-based CBT program to prevent depression and teach coping skills and can be provided with or without clinician guidance, it was developed by by researchers at the Australian National University in 2013 (www.moodgym.anu.edu.au). In this study MoodGYM was entirely self-directed. The program teaches key components of CBT for depression in five modules: Feelings, Thoughts, Unwrapping, De-stressing, and Relationships as shown in (Table 1). Each module contains exercises to be completed during the module, homework to be completed during the week, and a workbook to record progress throughout the program. The modules are designed to be completed in order, with each module estimated to take 30-45 minutes to complete, with 28 exercises and 13 quizzes in total across all modules [33]. 
Table 1. Modules of MoodGYM (www.ehubhealth.com)

\begin{tabular}{|ll|}
\hline Modules & Descriptions \\
\hline Feelings & $\begin{array}{l}\text { The Feelings module focuses on the basic tenets of CBT by highlighting connections } \\
\text { between events, thoughts, feelings, and behaviours. Those who will be using the } \\
\text { program will be introduced to six characters that have unique thought patterns and } \\
\text { feelings, some adaptive and some maladaptive. }\end{array}$ \\
\hline Unwarping & $\begin{array}{l}\text { The second module's basis is Thoughts, teaching users how to challenge what the } \\
\text { program identifies as their 'warped thoughts', by exploring the reasons behind their } \\
\text { thinking. }\end{array}$ \\
\hline $\begin{array}{l}\text { The third module is Unwarping. It has a total of ten exercises, which is the most any } \\
\text { module contains. These exercises aim to attack distorted thinking and build self- } \\
\text { esteem. One part of this module offers techniques on how to address the areas of } \\
\text { the vulnerability identified by the Warpy Thoughts Quiz. }\end{array}$ \\
$\begin{array}{l}\text { The fourth module is Distressing, which centres around recognizing the different } \\
\text { sources of stress and learning how to deal with them. An exercise known as 'Life } \\
\text { Whacks' consists of major stressful events, such as losing someone who is } \\
\text { important or having a conflict with parents. This module also focuses on relaxation } \\
\text { programs. }\end{array}$ \\
\hline $\begin{array}{l}\text { Relationships } \\
\text { The fifth module, Relationships, focuses on emotional and behavioural reactions to } \\
\text { relationship breakups and offers more ways to argue against related distorted } \\
\text { thoughts. }\end{array}$ \\
\hline
\end{tabular}

\section{Procedure}

Academic advisors sent invitation emails and participant information sheets explaining the study to all students scheduled to attend a seminar to address their poor academic performance in the previous semester (GPA < 2.0). Students who self-reported in the previous two weeks that they had felt that down, depressed, or hopeless, or had had little interest or pleasure in doing things, were invited to take part in the study by emailing the researcher. The academic advisor reminded students about the study invitation during the scheduled remedial class. All participants who contacted the researcher within the study timeframe (September 2019) were sent a link to the baseline survey. All parts of this study were conducted online, and data at baseline and eight-week follow-up were collected via anonymous surveys hosted by JISC Online Surveys. Students were able to re-read the participant information sheet before completing the online consent form. Participants were asked to create their own unique study ID code by providing their birthday and the last three digits of their mobile phone number. This personal ID was used to link baseline and follow-up data. Participants then completed the baseline survey, which included demographics, GPA, number of attendance warnings in the previous semester, previous help-seeking for mental health problems, and the HADS. At the end of the survey, students were thanked for their time and asked to click on a second survey link, which allowed them to enter their email address in order to receive a link and user code for MoodGYM. They were also provided with information regarding the University Counselling Centre if they felt they needed any further support. For those who provided their emails, the researcher sent them an email with instructions and user code for accessing MoodGYM. All participants 
were emailed a reminder about using MoodGYM four weeks after entering the study. Eight weeks after the baseline survey, they were emailed a link to the follow-up survey, which collected data on their GPA, number of attendance warnings in the current semester and the HADS. The follow-up survey also included text boxes to allow participants to comment on the positive and negative aspects of MoodGYM. A reminder email was sent to all participants two weeks after the follow-up survey email.

\section{Data analysis}

Data from the baseline and follow-up surveys were imported into SPSS (version 26). After cleaning and checking the data, HADS and GPA scores were compared pre- and post-intervention, using appropriate paired statistics. Univariate correlations and regression analysis were also used to explore the relationship between self-reported MoodGYM use and changes in GPA. A repeated-measures ANOVA with group (historic control/ intervention) as the independent factor, time of testing as the within-subjects factor and GPA score as the dependent variable was conducted to look for group by time interactions in GPA score. Descriptive statistics were used to explore the acceptability and usability of MoodGYM. Content analysis was used to classify and group the participants' responses to the open-ended question for evaluating MoodGYM.

\section{Results}

Fifty consecutive respondents ( 36 female and 14 male) completed the baseline survey and received access to the MoodGYM program. Of the 50 students, 47 accessed the program, and 44 completed the post-intervention survey and formed the study sample (88.0\% follow-up). Mean participant age was 20.7 years $(S D=1.55$; range $18-24)$ and the majority were female $(72 \%)$ (Table 2$)$. At baseline, there were no detectable differences in demographic characteristics or baseline GPA, depressive symptoms or number of attendance warnings between responders at follow-up (Intervention group, $n=44$ ) and non-responders at follow-up $(n=6)$ or between the intervention group responders at follow-up and the historic control group $(n=19)$ (all $p=>0.05)$. However, non-responders were substantially and significantly more anxious at baseline than responders $(\mathrm{M}=11.00, \mathrm{SD}=3.63, n=6$, vs. $\mathrm{M}=7.61, \mathrm{SD}=2.57, n=44 p=0.006, d=$ 1.2). There was no difference in baseline anxiety between the Intervention group and historic control.

Table 2. Demographic characteristics of the sample and comparison groups at baseline. 


\begin{tabular}{|c|c|c|c|}
\hline Variables & $\begin{array}{l}\text { Intervention group } \\
(n=44)\end{array}$ & $\begin{array}{l}\text { Non-responders } \\
(n=6)\end{array}$ & $\begin{array}{l}\text { Historic control group } \\
(n=19)\end{array}$ \\
\hline \multicolumn{4}{|l|}{ Gender } \\
\hline Male & $13(29.5 \%)$ & $1(16.7 \%)$ & $6(31.6 \%)$ \\
\hline Female & $31(70.5 \%)$ & $5(83.3 \%)$ & $13(68.4 \%)$ \\
\hline \multicolumn{4}{|l|}{ Age } \\
\hline $18-20$ & $19(43.2 \%)$ & $4(66.6 \%)$ & $8(42.1 \%)$ \\
\hline $21-24$ & $23(52.3 \%)$ & $2(33.3 \%)$ & $11(57.9 \%)$ \\
\hline Mean age (SD) & $20.7(1.46)$ & $20.4(1.95)$ & $20.00(1.84)$ \\
\hline \multicolumn{4}{|l|}{ Marital status } \\
\hline Married & $4(9.1 \%)$ & $2(33.3 \%)$ & $2(10.5 \%)$ \\
\hline Single & $40(90.9 \%)$ & $4(66.6 \%)$ & 17 (89.5\%) \\
\hline \multicolumn{4}{|l|}{ Year of study } \\
\hline $2^{\text {nd }}$ year & $24(54.5 \%)$ & $4(66.6 \%)$ & $12(63.2 \%)$ \\
\hline $3^{\text {rd }}$ year & $18(40.9 \%)$ & $2(33.3 \%)$ & $6(31.6 \%)$ \\
\hline $4^{\text {rd }}$ year & $2(4.5 \%)$ & 0 & $1(5.3 \%)$ \\
\hline \multicolumn{4}{|l|}{ Department of study } \\
\hline Business & $22(50.0 \%)$ & $2(33.3 \%)$ & $7(36.9 \%)$ \\
\hline Humanities \& Social Sciences & $2(4.5 \%)$ & 0 & $2(10.5 \%)$ \\
\hline Technological Innovation & $6(13.6 \%)$ & 0 & $3(15.8 \%)$ \\
\hline $\begin{array}{l}\text { Communication \& Medical } \\
\text { Sciences }\end{array}$ & $8(18.2 \%)$ & $2(33.3 \%)$ & $4(21.0 \%)$ \\
\hline Natural Health Sciences & $6(13.6 \%)$ & $2(33.3 \%)$ & $3(15.8 \%)$ \\
\hline
\end{tabular}

\section{Help seeking}

Prior to using MoodGYM, nearly half of the students reported seeking help for their mental health from their friends ( $n=19,43.2 \%)$ and a quarter of them had sought help from internet sources $(n=11,25.0 \%)$, Two participants (4.5\%) sought help from family, four (9.1\%) had sought help from their university tutor and only three $(6.8 \%)$ had sought help from university counselling services. Five students $(11.4 \%)$ had not sought any help for their mental health. 


\section{Depression and anxiety before and after MoodGYM}

A paired-samples t-test found a significant reduction in HADS-D scores post-intervention compared to baseline $(t(43)=3.07, p=0.004, d=0.5$ ), indicating a significant reduction in depressive symptoms (Table 3 ). The proportion of participants scoring above the cut-off for depression caseness from $77.2 \%$ to $27.3 \%$ ( $n=34$ to $n=12 ;$ McNemar $=p<0.001)$. There was also a decrease in HADS-A scores post-intervention ( $t$ $(43)=5.67, p \leq .001, d=1.1$ ), indicating a considerable reduction in anxiety symptoms. The proportion of students scoring above the cut-off for anxiety caseness fell from $50 \%$ to $11.4 \%$ ( $n=22$ to $n=5$; McNemar $=p<0.001$ ).

Table 3. Participants' depression and anxiety levels before and after MoodGYM

\begin{tabular}{|lll|}
\hline & $\begin{array}{l}\text { Pre-intervention } \\
(\mathrm{n}=44)\end{array}$ & $\begin{array}{l}\text { Post-intervention } \\
(\mathrm{n}=44)\end{array}$ \\
\hline HADS-D Group & $10(22.7 \%)$ & $32(72.7 \%) \star \star \star$ \\
Normal & & $5(11.4 \%)$ \\
\hline Borderline & $30(68.2 \%)$ & $7(15.9 \%)$ \\
\hline Clinical & $4(9.1 . \%)$ & $6.64(3.72)$ \\
\hline HADS-D & $8.32(1.72)$ & \\
Mean (SD) & & $39(88.6 \%) \star \star \star$ \\
\hline HADS-A group & & $2(4.6 \%)$ \\
\hline Normal & $22(50.0 \%)$ & $3(6.8 \%)$ \\
\hline Borderline & $18(40.9 \%)$ & $4.77(2.67)$ \\
\hline Clinical & $4(9.1 \%)$ & \\
\hline HADS-A & $7.61(2.57$ & \\
Mean (SD & & \\
\hline
\end{tabular}

Note: ${ }^{*} \mathrm{p} \leq 0.05,{ }^{\star \star} p \leq 0.01,{ }^{\star \star \star} \mathrm{p} \leq 0.001$

\section{Academic performance before and after MoodGYM}

Student GPA at pre-intervention ranged from 0.33-1.90, with a mean of $1.55(\mathrm{SD}=0.32)$. There was a significant increase in GPA at post-intervention $(t(43)=-9.26, p \leq 001 d=1.3)$, reflecting substantial improvement in academic performance after using MoodGYM (Table 4). At pre-intervention, all students in the intervention group had a GPA below 2.0 indicating academic weakness. After using MoodGYM, 19 $(43.2 \%)$ students had a GPA of 2.0 or above, thereby moving out of the academic warning zone. 
Half the sample had received at least one attendance warning $(n=22,50.0 \%)$ during the pre-intervention period; however, the number of participants who received attendance warnings decreased by nearly half after using MoodGYM (8 week intervention) ( $n=11,22.0 \%)$, with a significant reduction in the number of attendance warnings between pre- and post-intervention $(Z=-2.66, p=0.008, d=0.6)$ (Table 4).

Table 4. Academic outcomes pre- and post-intervention and in historic control group.

\begin{tabular}{|c|c|c|c|c|}
\hline \multirow[t]{2}{*}{ Intervention group $(n=44)$} & & \multicolumn{3}{|c|}{$\begin{array}{l}\text { Historic control } \\
(n=19)\end{array}$} \\
\hline & Baseline & Follow-up & Baseline & Follow-up \\
\hline Mean GPA (SD) & $\begin{array}{l}1.54 \\
(0.33)\end{array}$ & $1.99(0.33)$ & $1.56(0.39)$ & $1.75(0.51)$ \\
\hline $\begin{array}{l}\text { Mean number of attendance warnings } \\
\text { (SD) }\end{array}$ & $\begin{array}{l}1.05 \\
(0.75)\end{array}$ & $\underset{* \star}{0.61}(0.78)$ & $1.17(1.09)$ & $1.21(1.08)$ \\
\hline
\end{tabular}

Note: ${ }^{*} p \leq 0.05,{ }^{\star *} p \leq 0.01$

\section{Academic performance outcomes compared to historic control}

At baseline, the intervention group $(n=44)$ and the comparison group $(n=19)$ had similar GPAs and number of attendance warnings. A repeated-measures ANOVA with group (intervention/historic control) as the between-subjects factor and time found a significant time by group interaction $(F=5.96$, $d f=1.61, p=0.018)$. At follow-up, the intervention group had significantly higher GPAs compared to historic control $(t(61)=2.22, p=0.030 d=0.6)$ and fewer attendance warnings $(Z=-2.10, p=0.036 d=$ 0.7) (Table 4).

\section{Completed MoodGYM modules and outcome improvements}

All students in the Intervention group reported completing at least two modules, with nearly half of them reporting completing all five modules (mean completed $=3.75, \mathrm{SD}=1.52, n=44$ ). Almost all participants completed the Feelings module ( $n=42,95.5 \%)$, followed by Thoughts (36, 81.8\%), Unwrapping (34, 77.3\%), De-stressing (29,65.0\%), and Relationships (24,54.0\%). There was a significant positive correlation between GPA improvement and the number of modules completed $\left(r_{s}=0.388, n=44, \mathrm{p}=\right.$ 0.009). Completing more modules was also associated with a greater reduction in anxiety scores $\left(r_{s}=\right.$ $0.348, n=44, p=0.020$ ); however, no relationship was found between changes in depression scores and number of completed modules.

Regression analysis (entry method) with the difference between pre and post-intervention GPAs as the dependent variable (higher scores indicating greater GPA improvement), and baseline anxiety scores, baseline depression scores, completed MoodGYM modules, and improved attendance as the independent variables, found that greater improvement in GPA was associated with a higher number of completed 
MoodGYM modules $(\beta=.392, p=0.005)$ and improved attendance $\left(\beta=.388, p=0.007 ;\right.$ total adjusted $r^{2}=$ 0.35) (Table 5).

Table 5. Regression analysis for depression, anxiety, completed MoodGYM modules, attendance, and GPA pre- and post-intervention

\begin{tabular}{|lllll|}
\hline & \multicolumn{5}{c|}{ Post-intervention GPA } \\
\hline & $B$ & SE B & $B$ & $T$ \\
\hline Depression scores (pre-intervention) & .024 & .028 & .125 & .837 \\
\hline Anxiety scores (pre-intervention) & -.022 & .018 & -.171 & -1.194 \\
& & & & \\
\hline Total number of modules completed. & & & & \\
& .083 & .028 & .392 & 2.985 \\
\hline Improved attendance & & & & \\
\hline
\end{tabular}

Dependent Variable: Semester GPA (Post-intervention - Pre-intervention)

Note: ${ }^{*} p=<0.05,{ }^{\star \star} p=<0.01$

\section{Evaluation of MoodGYM}

The time period over which the participants used MoodGYM ranged from 2 weeks to 8 weeks; however, the highest proportion of students used the program for more than 4 weeks $(n=16,36.4 \%)$ (Table 6).

Table 6. Number of weeks students spent completing MoodGYM modules

\begin{tabular}{|ll|}
\hline Number of weeks & Number of students (\%) \\
\hline Two weeks & $4(9.0 \%)$ \\
\hline Three weeks & $5(11.4 \%)$ \\
\hline Four weeks & $9(20.5 \%)$ \\
\hline Five to eight weeks & $16(36.4 \%)$ \\
\hline Not sure about time spent & $10(22.7 \%)$ \\
\hline
\end{tabular}


Participant ratings of MoodGYM were generally either overwhelmingly positive or neutral. Most students ( $n=31,70.5 \%$ ) rated MoodGYM as good or very good, and 35 (79.6\%) found it very or slightly helpful. More than half ( $n=26,59.1 \%)$ found MoodGYM easy or very easy to use, and $33(75.0 \%)$ stated they would recommend it to a friend or family member.

Students were asked through an open-ended question to summarise how MoodGYM was helpful to them, and $70.5 \%(n=31)$ responded. Content analysis found that $20(45.5 \%)$

students reported that MoodGYM was helpful in reducing anxiety and depressive symptoms by teaching them different coping skills and strategies to address their negative thoughts. From the 20 students; four mentioned that the program helped them reflect on themselves more by completing different exercises and conducting self-assessments at the end of each module; five students stated that MoodGYM increased their knowledge about mental health; one student specifically suggested that it was a very good tool to deal with self-criticism and ten students mentioned that MoodGYM helped them acquire new self-help techniques and strategies to deal with their negative thoughts and change their daily routines by implementing some of the exercises. For example, one student stated 'MoodGYM helped me to deal with my worrying thoughts and to understand why I get them very often'.

Eight (18.2\%) students found MoodGYM helpful, but felt the modules were too long and time-consuming and two students (4.6\%) felt that MoodGYM was unhelpful, due to its lack of clarity and complexity. For example, one student was not able to understand the purpose of some assessments, stating, 'In all, I don't think it was clear enough'.

\section{Discussion}

In the present study, students with poor academic achievement who self-reported symptoms of low mood demonstrated significant and substantial improvements in GPA ( $d=1.3)$ and attendance $(d=0.6)$ after using an online CBT-based intervention (MoodGYM). Comparison with a previous cohort of students with low GPAs found a significant time by group interaction, with the MoodGYM group having very similar GPA scores to the historic control group at baseline, but significantly higher GPA scores at the equivalent follow-up point. Attendance also improved compared to control. Level of MoodGYM usage and improved attendance independently predicted improvement in GPA. MoodGYM was also associated with significant reductions in depressive and anxiety symptoms and there were substantial reductions in the proportion of students scoring above the cut-off for anxiety and depression caseness. MoodGYM was generally positively evaluated by students, and most participants suggested they would recommend the program to friends or family members.

Although this study lacked a control group for the substantial improvement in depressive and anxiety symptoms, the findings are in line with previous research using MoodGYM with student populations. A study of female secondary school students $(n=157)$ used MoodGYM in comparison with the school's standard development activities. The results suggested that MoodGYM was associated with a significantly decreased rate of self-reported depressive symptoms, compared to the usual curriculum, and 
the effect of MoodGYM was more significant at after 20 weeks [34]. A recent study exploring the prevention and treatment of major depressive disorder among high-risk first- and second-year university students [35] compared MoodGYM with two other internet-based preventative programs: attentional bias modification and an active attentional control condition. Depressive symptoms were assessed at three points: baseline, post-intervention, and 4-month follow-up using the Depression Anxiety and Stress Scale 21 (DASS-21) depression scale and the Beck Depression Inventory II (BDI-II). The results indicated that MoodGYM was a more effective intervention at both the diagnostic and symptom levels than the other two interventions, with effect sizes for change in BDI-II and DASS-21 depression scale of $d=0.40$ and 0.51 , respectively, at four-month follow-up. These were similar to the effect size $(d=0.5)$ for change in HADS-D observed in the current study at eight weeks post-intervention. Conversely, Twomey and colleagues [36] found no significant improvements in anxiety or depressive symptoms in a clinical sample group with reported mental health issues using MoodGYM compared to a waitlist control group, despite showing some decrease in general psychological distress. However, the mean age of 35 in the intervention group was substantially older than the present sample and the follow-up rate was only $18 \%$.

In the present sample, $70.5 \%(n=31)$ considered MoodGYM to be an effective program for reducing depressive and anxiety symptoms and felt it enhanced their knowledge of mental health. This is consistent with a study by Farrer and colleagues [19], which suggested that CBT interventions can effectively reduce depressive symptoms and promote knowledge about effective strategies for dealing with depression and self-management. Lintvedt and colleagues [37] reported that an unguided intervention (MoodGYM) effectively improved depressive symptoms and negative automatic thoughts in a university population. The study had a high dropout rate $(62 \%$ of the participants responded postintervention). Linvedt and colleagues (37) in their study with University student experiencing depressive symptoms also found that participants who used MoodGYM were very satisfied, and $90 \%$ stated that they would recommend the program to others.

The results of the present study sample suggested a significant improvement in academic performance and a significant reduction in attendance warnings after using MoodGYM. To our knowledge, this is the first study to explore the potential of a CBT-based online intervention to improve academic outcomes. However, previous studies that used different online interventions reported some improvements in academic achievement. For example, a recent study by Viskovich and Pakenham [38] that explored the effectiveness of web-based Acceptance and Commitment Therapy (ACT) in promoting mental health in university students reported pre- to post-intervention improvements for the study's primary outcomesincluding academic performance-and these improvements were maintained throughout the follow-up period. The study used a four-week web-based ACT mental health promotion intervention called 'YOLO'. Academic performance was measured using a brief, 12-item self-report scale measuring facets of academic performance, including study habits, study motivation, and overall grades. It was not known which items contributed to the score, as the study used a factor analysis method; however, results indicated that the intervention improved participants' perceived performance, although objective performance was not assessed. 
In the present sample, the comparison group $(n=19)$ was from a recent cohort study conducted by Awadalla and colleagues [3], to explore the effects of depression and anxiety on academic performance among university students. Essentially, the historic control group was from the same sample population of the current study (i.e. same university and very similar sample characteristics). Like the intervention group the students in this group had GPAs below 2.0 at recruitment and were considered in the academic warning (failing) category. In comparison with the intervention group $(n=44)$, they had similar GPAs and attendance warnings at baseline; however, the comparison group (no-intervention) had significantly lower GPAs and more attendance warning at follow-up, demonstrating that the intervention group had better academic outcomes over a similar period.

There are many societal, attitudinal, and cultural reasons why university students with emotional difficulties may not seek professional help [39]. The present study findings show a gap in the proportion of students experiencing emotional difficulties and those who had sought professional help. For example, although $77 \%$ scored above the cut-off for depression caseness and nearly half of the intervention group had sought help from their friends, only three students had sought professional help. MoodGYM appears to offer an effective and acceptable method of increasing access to evidence based therapies.

In this study, out of the 47 students who accessed MoodGYM, half completed the all five modules. The results indicated that the students who completed more MoodGYM modules performed better academically than those who completed fewer modules. The findings support the importance of the dose of the intervention; however, the bias of self-report measures and the need to assess the fidelity of MoodGYM use should also be considered These findings are consistent with those of Calear and colleagues [40], who investigated the effects of adolescent adherence to MoodGYM in schools. They reported that participants who maintained high adherence to the intervention reported more substantial intervention effects six months post-intervention, compared with participants with low adherence.

In the current study more than half (59.1\%) of the students found MoodGYM easy to use, and threequarters of the students would recommend it to a family member or friend. This is in line with another study that evaluated MoodGYM in primary care patients with mild to moderate depression. The study reported that MoodGYM was rated positively by more than half of the participants and suggested that low non-adherence rates was a sign of positive evaluation indicating the intervention's acceptability [41]. The study concluded that significant improvements were found at two-month follow-up, and the level of satisfaction among the participants was high, as $90 \%$ reported they would recommend MoodGYM to others.

Even though most of the students in the present study found MoodGYM to be helpful and easy to access, some found the modules to be very long and time-consuming. These students may require additional therapist support. A systematic review by Knowles and colleagues [42] investigated qualitative studies exploring user experience with web-based therapies for anxiety and depression. The review included eight studies, of which six used CBT treatment, and the findings highlighted that web-based CBT interventions have shown more concern regarding improving access to therapy than on patient experience. The review 
suggested that considering the sensitivity and personalising the program's content to be more relevant to users could increase engagement and adherence. Furthermore, Neil and colleagues [43] proposed that internet-based interventions should precisely record user activity to accurately measure adherence. Estimating the time spent on modules is significant, in light of the fact that a user could also spend considerable time reading the modules but not complete the exercises, and yet still display some benefits from the program.

\section{Strengths and limitations}

This appears to be the first study to evaluate an online CBT-based intervention to support students with low mood and academic difficulties in the UAE. The study used a pre- and post-intervention design, which can be a valuable for providing preliminary evidence for intervention effectiveness. The fact that $88 \%$ of the students responded to the post-intervention survey supports the credibility of the study and its subsequent results. Furthermore, the scale used in this study for anxiety and depression (HADS) has been validated and shows good sensitivity and specificity. The HADS questionnaire has been validated in many languages, countries, and settings, including in the UAE [34]. Another strength of this study was the use of a historical control group for GPAs, recruited from the same university and showing data at the same timepoints during the previous year. The results of this pilot study constitute an important step towards further longitudinal studies to explore the effectiveness of online interventions in supporting academic progress for university students with mental health issues.

Some limitations of this study should be noted. Due to the small sample size and short follow-up period, the results reflect only a short 'window of time', and within a limited university population. Thus, with more students and an extended follow-up period, the results could be more accurate and less biased, thereby more reliably estimating the intervention's benefits. Other limitations are that this study was not a randomised trial, had no control group for depressive and anxiety symptoms and used self-reported GPAs. Finally, limited qualitative data to support the quantitative findings, and the bias related to being in a study and the expectation to complete the survey, can produce inauthentic answers.

\section{Conclusions}

Results indicate that MoodGYM seems to be a convenient, acceptable and effective therapeutic intervention when targeted at academically struggling students with low mood. The observed improvements in mood, GPA and attendance suggest that MoodGYM may be a cost-effective way to overcome barriers to mental health support for academically struggling students. However, more research is needed to explore whether improvements are sustained and how MoodGYM can be best implemented within the curriculum.

\section{Abbreviations}

ACT Acceptance and Commitment Therapy 
CBT Cognitive Behavioural Therapy

GPA Grade Point Average

HADS Hospital Anxiety and Depression Scale

DASS-21 Depression Anxiety and Stress Scale 21

BDI-II Beck Depression Inventory II

ANOVA Analysis of Variance

UAE United Arab Emirates

\section{Declarations}

\section{Ethics approval and consent to participate}

Ethical approval was obtained from the Division of Psychiatry and Applied Psychology Ethics Subcommittee (reference number: 0397) and the Research Ethics Committee at Zayed University (ref ZU19_46_F). Participation was voluntary. Students provided the written consent online to participate in the two anonymous online surveys, which were linked by self-generated identifiers.

\section{Consent for publication}

Not applicable

\section{Availability of data and materials}

The datasets generated during and/or analysed during the current study are not publicly available [PhD study under progress] but are available from the corresponding author on reasonable request.

\section{Competing interests}

The authors declare they have no competing interests.

\section{Funding}

The project was funded through a PhD studentship awarded to SA by the Ministry of Higher Education and Zayed University, United Arab Emirates.

\section{Authors' contributions}

SA, CG and EBD designed the study protocol and surveys. SA analysed the data under CG's supervision. SA prepared the first draft of the paper and CG and EBD reviewed subsequent drafts. All authors read and approved the final manuscript. 
Acknowledgements

SA would like to acknowledge and thank all the students who took part in this study, and the academic advisors who helped in recruiting the students for this study.

\section{References}

1. Ibrahim A, Kelly S, Adams C, Glazebrook C. A systematic review of studies of depression prevalence in university students. J Psychiatr Res. 2013; 47:391-400.

2. Abu Ruz M, Al-Akash $\mathrm{H}$, Jarrah S. Persistent (anxiety and depression) affected academic achievement and absenteeism in nursing students. Open Nurs J. 2018; 12:171-9.

3. Awadalla S, Davies E, Glazebrook C. A longitudinal cohort study to explore the relationship between depression, anxiety and academic performance among Emirati university students. BMC Psychiatry. 2020;20(1).

4. DeRoma VM, Leach JB, Leverett JP. The relationship between depression and college academic performance. Coll Stud J. 2009; 43:325-35.

5. Dardas L, Simmons L. The stigma of mental illness in Arab families: a concept analysis. J Psychiatr Ment Health Nurs. 2015; 22:668-79.

6. Harrer M, Adam S, Baumeister H, Cuijpers P, Karyotaki E, Auerbach R, et al. Internet interventions for mental health in university students: a systematic review and meta-analysis. Int J Methods Psychiatr Res. 2018;2:e1759.

7. January J, Madhombiro M, Chipamaunga S, Ray S, Chingono A, Abas M. Prevalence of depression and anxiety among undergraduate university students in low- and middle-income countries: a systematic review protocol. Syst Rev. 2018; 7:57.

8. Grist R, Croker A, Denne M, Stallard P. Technology delivered interventions for depression and anxiety in children and adolescents: a systematic review and meta-analysis. Clin Child Fam Psychol Rev. 2018; 22:147-71.

9. Farrer L, Gulliver A, Chan J, Batterham P, Reynolds J, Calear A, et al. Technology-based interventions for mental health in tertiary students: systematic Review. JMIR. 2013;15: e101.

10. Davies E, Morriss R, Glazebrook C. Computer-delivered and web-based interventions to improve depression, anxiety, and psychological well-being of university students: a systematic review and meta-analysis. JMIR. 2014;16: e130.

11. Barrable A, Papadatou-Pastou M, Tzotzoli P. Supporting mental health, wellbeing and study skills in Higher Education: an online intervention system. International Journal of Mental Health Systems. 2018;12(1).

12. Bolinski F, Boumparis N, Kleiboer A, Cuijpers P, Ebert D, Riper $H$. The effect of e-mental health interventions on academic performance in university and college students: A meta-analysis of randomized controlled trials. Internet Interv. 2020; 20:100321. 
13. Gaudiano B. Cognitive-behavioural therapies: achievements and challenges. Evid Based Ment Health. $2008 ; 11: 5-7$.

14. Lopez M, Basco M. Effectiveness of cognitive behavioral therapy in public mental health: comparison to treatment as usual for treatment-resistant depression. Admin Policy Ment Health. 2014; 42:87-98.

15. Sweetland A, Oquendo M, Sidat M, Santos P, Vermund S, Duarte $C$, et al. Closing the mental health gap in low-income settings by building research capacity: perspectives from Mozambique. Ann Glob Health. 2014; 80:126.

16. Anderson J, Howarth E, Vainre M, Jones P, Humphrey A. A scoping literature review of service-level barriers for access and engagement with mental health services for children and young people. Child Youth Serv Rev. 2017; 77:164-76.

17. Gratzer D, Khalid-Khan F. Internet-delivered cognitive behavioural therapy in the treatment of psychiatric illness. CMAJ. 2015; 188:263-72.

18. Christensen H, Griffiths K, Groves C, Korten A. Free range users and one hit wonders: community users of an internet-based cognitive behaviour therapy program. Aust N Z J Psychiatry. 2006; 40:5962.

19. Farrer L, Christensen H, Griffiths K, Mackinnon A. Web-based cognitive behavior therapy for depression with and without telephone tracking in a national helpline: secondary outcomes from a randomized controlled trial. JMIR. 2012;14: e68.

20. Twomey C, O'Reilly G. Meta-analysis looks at effectiveness of MoodGYM programme in computerised cognitive behavioural therapy. BMJ. 2016;354: i4221.

21. Christensen $H$, Griffiths K, Korten A. Web-based Cognitive behavior therapy: analysis of site usage and changes in depression and anxiety scores. JMIR. 2002;4: e3.

22. McDermott R, Dozois D. A randomized controlled trial of Internet-delivered CBT and attention bias modification for early intervention of depression. Journal of Experimental Psychopathology. 2019;10(2):204380871984250.

23. Kroenke K, Spitzer R, Williams J, Löwe B. An ultra-brief screening scale for anxiety and depression: the PHQ-4. Psychosomatics. 2009; 50:613-21.

24. Faul F, Erdfelder E, Lang A, Buchner A. G*Power 3: A flexible statistical power analysis program for the social, behavioral, and biomedical sciences. Behavior Research Methods. 2007;39(2):175-191.

25. Zigmond A, Snaith R. The Hospital Anxiety and Depression Scale. Acta Psychiatr Scand. 1983; 67:361-70.

26. Stern A. The Hospital Anxiety and Depression Scale. Occup Med (London). 2014;64:393-4.

27. Bjelland I, Dahl A, Haug T, Neckelmann D. The validity of the Hospital Anxiety and Depression Scale. An updated literature review J Psychosom Res. 2002; 52:(2):69-77.

28. Andrews B, Hejdenberg J, Wilding J. Student anxiety and depression: comparison of questionnaire and interview assessments. J Affect Disord. 2006; 95:29-34. 
29. El-Rufaie O, Absood G. Validity study of the Hospital Anxiety and Depression Scale among a group of Saudi patients. Br J Psychiatry. 1987; 151:687-8.

30. Malasi T, Mirza I, El-Islam M. Validation of the Hospital Anxiety and Depression Scale in Arab patients. Acta Psychiatr Scand. 1991; 84:323-6.

31. El-Rufaie O, Absood G. Retesting the validity of the Arabic version of the Hospital Anxiety and Depression (HAD) scale in primary health care. Soc Psychiatry Psychiatr Epidemiol. 1995; 30:26-31.

32. Terkawi A, Tsang S, AlKahtani G, Al-Mousa S, Al Musaed S, AlZoraigi U, et al. Development and validation of Arabic version of the Hospital Anxiety and Depression Scale. Saudi J Anaesth. 2017; $11: 11$.

33. Christensen $\mathrm{H}$. Delivering interventions for depression by using the internet: randomised controlled trial. BMJ. 2004; 328:265.

34. O'Kearney R, Kang K, Christensen H, Griffiths K. A controlled trial of a school-based internet program for reducing depressive symptoms in adolescent girls. Depress Anxiety. 2009; 26:65-72.

35. McDermott R, Dozois D. A randomized controlled trial of Internet-delivered CBT and attention bias modification for early intervention of depression. J Exp Psychopathol. 2019; 10:204380871984250

36. Twomey C, O'Reilly G, Byrne M, Bury M, White A, Kissane S, et al. A randomized controlled trial of the computerized CBT programme, MoodGYM, for public mental health service users waiting for interventions. Br J Clin Psychol. 2014; 53:433-50.

37. Lintvedt O, Griffiths K, Sørensen K, Østvik A, Wang C, Eisemann M, et al. Evaluating the effectiveness and efficacy of unguided internet-based self-help intervention for the prevention of depression: a randomized controlled trial. Clin Psychol Psychother. 2011; 20:10-27.

38. Viskovich S, Pakenham K. Randomized controlled trial of a web-based Acceptance and Commitment Therapy (ACT) program to promote mental health in university students. J C Psychol. 2019; 76:92951.

39. Heath P, Vogel D, Al-Darmaki F. Help-Seeking Attitudes of United Arab Emirates Students. The Counseling Psychologist. 2016;44(3):331-352.

40. Calear A, Christensen H, Mackinnon A, Griffiths K. Adherence to the MoodGYM program: outcomes and predictors for an adolescent school-based population. J Affect Disord. 2013; 147:338-44.

41. Høifødt R, Lillevoll K, Griffiths K, Wilsgaard T, Eisemann M, Waterloo K, et al. The clinical effectiveness of web-based cognitive behavioral therapy with face-to-face therapist support for depressed primary care patients: randomized controlled trial. JMIR. 2013;15:e153.

42. Knowles S, Toms G, Sanders C, Bee P, Lovell K, Rennick-Egglestone S, et al. Qualitative metasynthesis of user experience of computerised therapy for depression and anxiety. PLOS ONE. 2014;9: e84323.

43. Neil A, Batterham P, Christensen H, Bennett K, Griffiths K. Predictors of adherence by adolescents to a cognitive behavior therapy website in school and community-based settings. JMIR. 2009;11: e6. 